\title{
A NOTE ON COHERENT RINGS OF DIMENSION TWO
}

\author{
ZHAO YICAI
}

(Communicated by Louis J. Ratliff, Jr.)

\begin{abstract}
It is shown that a commutative coherent domain cannot have $\mathrm{Ng}$ dimension 2 and a commutative coherent regular ring of $\mathrm{Ng}$ dimension 2 cannot have finite indecomposable decomposition.
\end{abstract}

\section{INTRODUCTION}

Throughout, $R$ will denote a commutative ring with $1 \neq 0$. In $[4,3]$, $\mathrm{H}$. K. Ng first introduced the finitely presented dimensions of modules and commutative rings to investigate coherent rings. Some interesting results were obtained. In order to avoid confusion we rename this dimension $\mathrm{Ng}$ dimension (see [1, p. 221]).

A ring $R$ is called an $(a, b, c)$-ring if w.gl. $\operatorname{dim} R=a, \operatorname{gl} \operatorname{dim} R=b$, and $\mathrm{Ng} \operatorname{dim} R=c$. it was asserted in [3] that a coherent domain of global dimension 2 must be $(1,2,3)-,(2,2,0)-,(2,2,2)$ - or $(2,2,3)$-ring. In this paper, we show that a coherent domain cannot have $\mathrm{Ng}$ dimension 2 , and hence there is no $(2,2,2)$-coherent domain. It is also shown that a coherent regular ring of $\mathrm{Ng}$ dimension 2 cannot have finite indecomposable decomposition.

Lemma. Let $R$ be a commutative ring. The following are equivalent for $R$ modules:

(1) Projective ideals are finitely generated.

(2) A projective module with finitely-generated localizations is finitely generated. (See [5, Theorem 2.1].)

Theorem. A coherent domain cannot have $\mathrm{Ng}$ dimension 2.

Proof. Let $R$ be a coherent domain. If $\mathrm{Ng} \operatorname{dim} R=2$, then there is an ideal $I$ with $\operatorname{Ng} \operatorname{dim} I=1$. Hence there exists an exact sequence $0 \rightarrow K \rightarrow F \rightarrow I \rightarrow 0$ such that $F$ is a nonfinitely-generated projective $R$-module and $K$ is a finitelygenerated $R$-module. By [4, Proposition 1.7] and [3, Proposition 2.4], $R_{P}$ is a Noetherian ring for every prime ideal $P$ of $R$, and hence $I_{P}$ is finitely generated. Notice that $K_{P}$ is also finitely generated. It follows that $F_{P}$ is finitely generated for every prime ideal $P$. Since $R$ is a domain, every projective ideal is invertible and hence is finitely generated. By the lemma, $F$ is finitely generated, a contradiction. Therefore $\operatorname{Ng} \operatorname{dim} R \neq 2$.

Received by the editors October 12, 1990 and, in revised form, January 22, 1991.

1991 Mathematics Subject Classification. Primary 13D05. 
Corollary 2. Let $R$ be a domain of global dimension 2. Then $R$ must be a (1, 2, 3)-, (2, 2, 0)- or (2, 2, 3)-ring. Moreover, a (1, 2, 3)-domain is a Prüfer domain, $a(2,2,0)$-domain is a Noetherian domain, and a (2, 2, 3)-domain is an integrally closed coherent domain.

Proof. First of all, $R$ is a coherent ring. Indeed, suppose $I$ is a finitelygenerated ideal of $R$. There exists an exact sequence $0 \rightarrow K \rightarrow F \rightarrow I \rightarrow 0$ where $F$ is finitely generated projective and $K$ is projective. For any prime ideal $P$ of $R, K_{P}$ is finitely generated since $R_{P}$ is coherent. By the lemma, $K$ is finitely generated. It follows that $R$ is coherent. On the other hand, $R_{P}$ is a GCD domain by [7, Theorem 2.2], and hence $R$ is an integrally closed domain by [4, Theorem 50]. The proof can be completed from Theorem 1, [4, Theorem 3.4], and [2, Theorem 64].

Remark. (2, 2, 3)-domains do exist (see [6, p. 383]).

A ring $R$ is called a regular ring if every finitely-generated ideal of $R$ has finite projective dimension.

Theorem 3. Let $R$ be a coherent regular ring. If $R$ has $N g$ dimension 2 , then $R$ cannot have a finite indecomposable decomposition.

Proof. Let $P$ denote an arbitrary prime ideal of $R$. By [1, Corollary 6.2.4], $R_{P}$ is a domain. $a R_{P}$ is a flat ideal of $R_{P}$ for every nonzero element $a$ of $R$, and hence $a R$ is flat. Since $a R$ is finitely presented, $a R$ is projective, which shows that the exact sequence $0 \rightarrow \operatorname{ann}(a) \rightarrow R \rightarrow a R \rightarrow 0$ splits. Hence there exists an ideal $I$ such that $R=\operatorname{ann}(a) \oplus I$. If $R$ is indecomposable, then it follows that $\operatorname{ann}(a)=0$; that is, $R$ is a domain, and $\operatorname{Ng} \operatorname{dim} R=2$. By Theorem 1 , this is a contradiction. Hence $R$ cannot be indecomposable. Now assume that $R$ has a finite indecomposable decomposition, $R=R_{1} \oplus R_{2} \oplus \cdots \oplus R_{n}$, say. By [4, Theorem 2.11], there is an $R_{i}$ such that $\operatorname{Ng} \operatorname{dim} R_{i}=\operatorname{Ng} \operatorname{dim} R=2$. On the other hand, $R_{i}$ is an indecomposable coherent regular ring. From the above argument, $\operatorname{Ng} \operatorname{dim} R_{i} \neq 2$, a contradiction.

Corollary 4. Let $R$ be a coherent regular ring and let $\mathrm{Ng} \operatorname{dim} R=2$. Suppose $e$ is a nonzero idempotent of $R$. Then $e$ is a finite sum of pairwise orthogonal primitive idempotents if and only if $e R$ is a Noetherian ring.

In particular, $e$ is a primitive idempotent if and only if $e R$ is a Noetherian domain.

Proof. Suppose $e=e_{1}+\cdots+e_{n}$, where every $e_{i}$ is primitive idempotent and $e_{i} e_{j}=0$ for $i \neq j$. Then $e R=e_{1} R \oplus \cdots \oplus e_{n} R$ is a finite direct sum of indecomposable rings. On the other hand, $e R$ is a coherent regular ring and $\mathrm{Ng} \operatorname{dim} e R \leq 2$. By Theorem 3, $\mathrm{Ng} \operatorname{dim} e R=0$. Therefore $e R$ is Noetherian.

The converse is clear.

\section{REFERENCES}

1. S. Glaz, Commutative coherent rings, Lecture Notes in Math., vol. 1371, Springer-Verlag, Berlin and New York, 1989.

2. I. Kaplansky, Commutative rings, Allyn and Bacon, Boston, MA, 1970.

3. H. K. Ng, Coherent rings of dimension 2, Proc. Amer. Math. Soc. 91 (1984), 518-521.

4. _ Finitely presented dimension of commutative rings and modules, Pacific J. Math. 113 (1984), 417-431. 
5. W. B. Vasconcelos, On projective modules of finite rank, Proc. Amer. Math. Soc. 22 (1969), 430-433.

6. __ The local rings of global dimension 2, Proc. Amer. Math. Soc. 35 (1972), 381-386.

7. __ The rings of dimension 2, Lecture Notes in Pure Appl. Math., vol. 22, Marcel Dekker, New York, 1976.

Department of Mathematics, Guangxi Teachers University, Guillin 541004, China 